\title{
The lightweight Delft Cylinder Hand, the first multi-articulating hand that meets the basic user requirements
}

\author{
Gerwin Smit, Dick H. Plettenburg, Frans C.T. van der Helm
}

\begin{abstract}
Rejection rates of upper limb prostheses are high (23-45\%). Amputees indicate that the highest design priority should be reduction of the mass of the prosthetic device. Despite all efforts, the mass of the new prosthetic hands is $35-73 \%$ higher than that of older hands. Furthermore current hands are thicker than a human hand, they operate slower and do not provide proprioceptive force and position feedback. This study presents the Delft Cylinder Hand, a body powered prosthetic hand which mass is $\mathbf{5 5 - 6 8 \%}$ lower than that of the lightest current prosthetic hands, operates faster, has an anthropomorphic shape, and provides proprioceptive force and position feedback. The hand has articulating fingers, actuated by miniature hydraulic cylinders. The articulating fingers adapt itself to the shape of the grasped object. Its functional scores are similar to that of current prosthetic devices. The hand has a higher mechanical performance than current body powered hands. It requires $49-162 \%$ less energy from the user and it can deliver a higher maximum pinch force $(30-60 \mathrm{~N})$.
\end{abstract}

Index Terms - Prosthetics, Artificial limbs, Prosthetic hand, Body Powered, Hydraulics.

\section{INTRODUCTION}

$\mathrm{R}$ eplacement of a missing hand by an artificial alternative remains one of the biggest challenges in prosthetic rehabilitation [1]. Although many different prosthetic terminal upper limb devices are available [2-4], around $27 \%$ of the amputees does not actively use its device and another 20\% stops wearing it at all [5]. There are various reasons for prosthesis abandonment; wearing discomfort (too heavy, too hot), too little added functionality, difficult or tiring to use, or the lack of sensory feedback [5]. The most important design priority is the device mass, which should be significantly reduced [6]. A hand prosthesis should at least meet the basic user demands, summarized by the words comfort, cosmetics and control; 'a patient wants and expects a prosthesis that looks naturally beautiful, that is comfortable to wear and that is easy to use' [7].

Gerwin Smit (contact author), Dick H. Plettenburg and Frans C.T. van der Helm, are with the Department of BioMechanical Engineering, Delft University of Technology, Mekelweg 2, 2628 CD Delft, The Netherlands (phone: +31-15 27 81688; e-mail: g.smit@tudelft.nl).
Currently two types of active prostheses are available; myoelectric and body-powered (BP) prostheses. In recent years new myoelectric hands have become available: the i-limb (www.touchbionics.com), the bebionic (www. bebionic.com), and the Michelangelo hand (www.ottobock.com). These hands provide a set of different grip patterns. Unlike other prosthetic hands, the fingers of the i-limb and the bebionic can articulate to provide an adaptive grip. Although these new hands provide more grip patterns, their additional functional value has not been proven yet [8], and the hands still remain too heavy (an average articulating hand weights $\sim 550$ gram, without battery and glove). A device mass of $\sim 350$ gram is already too heavy for amputees with a short residual arm [9].

The alternative to the myoelectric device is the BP hand or hook. The reported rejection rates of $\mathrm{BP}$ hands $(65 \%-80 \%)$ are significantly higher than that of BP hooks (32-51\%), or electric hands (17-41\%) $[6,10]$. A possible explanation of this low acceptance can be found in the limitations of the currently available BP hands. Although they have a lower mass $(\sim 350$ gram $)$ than electric hand they are still heavy. They produce a low pinch force $(\sim 15 \mathrm{~N})$ and require a high actuation force (61-131 N), due to their low mechanical efficiency $[11,12]$. Furthermore their shape is not closely anthropomorphic and they have rigid fingers, which do not adapt to the shape of the grasped object. This reduces the contact area between hand and object and limits the grasping functionality [13]. Despite these drawbacks, no improvements have been made to BP hands for decades $[11,12]$. If these matters could be resolved, a BP hand could offer several benefits over a myoelectric hand; the harness system of a BP hand offers proprioceptive force and position feedback to the user. Also a BP hand could be considerably lighter than a myoelectric hand, as no motors and batteries are required.

To increase the mechanical efficiency of a mechanism, mechanical linkages can be replaced by hydraulics. Hydraulics potentially offers an efficient way of energy transmission, as the friction losses in hydraulic systems are typically low. There have been various attempts to use hydraulics in upper limb prosthetics, in an effort to develop an 
efficient externally powered or body-powered terminal device. Several prototypes have used hydraulics to operate an externally powered prosthetic hand, e.g. Janovsky et al. [14], Broome et al. [15], Witte [16], Tobergte [17], Kato [18], Kargov et al. [19]. In other prototypes Goller et al. [20] and LeBlanc et al. [21] used a hydraulic transmission to operate a BP hook. There are no examples of the application of a hydraulic transmission in a BP hand in literature, nor in any commercially available BP or electric hand.

\section{PROBLEM}

Body-powered hands have the highest user rejection rate of all available terminal devices. They are heavy, require a high operation force from the user and then produce a low pinch force, due to mechanical inefficiencies. Furthermore their shape is not closely anthropomorphic and they have rigid fingers, which limits their grasping functionality.

\section{GOAL}

To develop and evaluate a lightweight prosthetic hand with articulating fingers and body-powered control, that meets the basic user demands. The hand should require less actuation energy than current body-powered hands (1.058 $-2.292 \mathrm{Nm}$ or J) and it should produce sufficient pinch force $(>30 \mathrm{~N})$ for a broad functional range. The hand will be evaluated by quantifying its mechanical performance by mechanical tests and its functional performance by functional user tests. The results will be compared to other prostheses described in literature.

\section{METHODS}

Requirements and design principles

A new lightweight articulating hand was designed, which has voluntary closing body-powered control. The requirements to which the hand had to comply are listed below. Various design principles were used to meet these requirements:

\section{- Cosmetic appearance}

The hand mechanism should fit inside a standard 7 $1 / 2$ " size anthropomorphic silicone cosmetic glove, e.g. the SGL5/E4 glove (RSL Steeper) or the 101L M2 Male glove (Regal Prosthesis Ltd.). Preferable a silicone glove should be used, as they are more compliant and have a lower hysteresis than PVC gloves [22]. In order to give the hand an anthropomorphic appearance, the shape of the mechanism should be adapted to the shape of the glove, instead of the other way around.

\section{- Mass}

In order to increase wearing comfort, the mass of the hand should be lowered [6]. Mechanism and glove should weigh less than a human hand (426 \pm

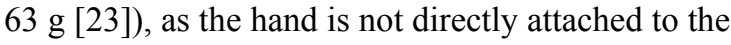
musculoskeletal system of the user and is therefore perceived as an external load. As a cosmetic glove weighs around $90 \mathrm{~g}$ [12] and one standard deviation is $63 \mathrm{~g}$ below the average mass of a human hand, the mass of the hand mechanism should be lower than $426 \mathrm{~g}-(63+90) \mathrm{g}=273 \mathrm{~g}$.

\section{- Actuation energy and pinch force}

The energy dissipation by the transmission should be minimized, to reduce the required input effort by the user and to produce a high pinch force $(>30 \mathrm{~N})$ to enable a broad range of activities [24]. A hydraulic system can transmit and distribute the energy and forces in an efficient way to multiple actuators, without the need of a whiffletree like mechanism with extra joints, with pulley cables or a linkage system. In this way extra friction can be avoided. Although the hand will have more DoF's than current BP hands, it should require less input energy than current hands (range of current hands: $1.058-2.292 \mathrm{Nm}$ or $\mathrm{J}[11]$ ), to enable easy and comfortable operation.

\section{- Control of the hand}

Control of the prosthetic hand should be fast, easy and intuitive. This can potentially be achieved by body-powered (BP) control. BP-control provides the user with direct proprioceptive force and position feedback, which enables precise and fast control of force as well as hand opening. This is a benefit over myoelectric control, which does not provide proprioceptive feedback. Furthermore BPcontrol requires no batteries and motors, which makes BP controlled prostheses potentially lighter. There are two modes of body-powered control, voluntary closing and voluntary opening control. For the new hand voluntary closing is preferred, as this provides the user with a direct and intuitive relation between actuation force and pinch force [25-27].

\section{- Articulating fingers}

To enable grasping and holding of a large variety of objects, the hand should be able to perform the two basic grip patterns: the precision or pinch grip, and the cylinder or power grip [28]. The pinch grip can be used for picking up small objects by means of force closure. The cylinder grip can be used to pick up larger objects, by means of force and form closure (the fingers wrap around the object). A pinch grip can be achieved with rigid fingers with one degree of freedom (DoF). To enable a power grip the fingers should have at least two DoF's, one in the MCP and one in the PIP joint. It is not necessary to have an extra DoF in the DIP joint, this joint can be fixed at an angle of $15^{\circ}$. This is the same angle that is used in case of arthrodesis surgery of the DIP joint [29]. As there is only one control signal available, to control the multiple DoF's of the hand, the hand is by definition underactuated [30, 31]. To obtain stable grasping in both pinch and power grasp tasks, without ejecting 
the grasped object, the torque ratio between $\mathrm{MCP}$ and PIP joint should be around 0.5 [32]. The thumb of the hand should be opposable to the fingers, to enable a pinch grip with one or two fingers. This could be done passively. The opening of the hand should be at least $70 \mathrm{~mm}$, to enable grasping of a broad range of objects.

\section{- Environmental influences}

The hand should be able to function in wet and dirty conditions, to enable a broad application and to guarantee a high reliability. Therefore no electronic components should be used, and the selected materials should be corrosion resistant.

\section{- Modular system}

The hand should be modular to enable easy maintenance and replacements of parts or modules by the prosthetist (e.g. one finger or one actuator). Modularity also provides the prosthetist with more freedom to customize the hand to an individual patient. The number of parts that can only be used for either a left or a right hand should be minimized [33].

\section{Hand prototype evaluation}

The mechanical performance of the constructed hand was evaluated in mechanical tests. The functional performance was assessed by functional user tests. Both tests used quantitative outcomes, to enable comparison to current available prosthetic devices and to past and future prototypes.

\section{- Mechanical evaluation}

A mechanical test bench measured the energy required to operate the hand and the energy that was dissipated by the hand. A load cell (Zemic: FLB3G$\mathrm{C} 3-50 \mathrm{~kg}-6 \mathrm{~B})$ measured the force that was required to operate the master cylinder. A displacement sensor (Schaevitz: LCIT 2000) measured the actuation displacements of the master cylinder. The required energy and the dissipated energy could be derived by integrating the measured forces along the measured displacements. A custom built pinch force load cell (thickness $=10 \mathrm{~mm}$ ) was used to measure the pinch force.

\section{-- Protocol for mechanical evaluation}

In the mechanical evaluation the hand was subjected to four different test protocols:

1. A full closing and opening cycle, without pinching (measured four times)

2. A full cycle of closing, opening and pinching an object (thickness $=10 \mathrm{~mm}$ ) with a pinch force of $15 \mathrm{~N}$ (measured four times).

3. Closing and pinching the pinch load cell (thickness $=10 \mathrm{~mm}$ ), until an actuation force of $100 \mathrm{~N}$ was reached.

4. Endurance was tested by closing, pinching $15 \mathrm{~N}$ and re-opening of the hand 100,000 times. Two sensors detected whether the index finger of the hand had reached sufficient pinch force and had fully opened during each cycle. When these criteria were not reached, the cycle discontinued and the setup was checked prior to continuation.

During test 1 and 2, the required input energy (work) and the energy dissipation (system hysteresis), which is a measure of the energy efficiency of the hand, were recorded and were compared to that of current hands. In test 3 the pinch force is measured as function of the actuation force. The characteristic was compared to that of other hands. The actuation force at which the pinch force starts building up should be as low as possible. Finally the hand prototype should pass test 4 , the endurance test, without the need of major repair.

\section{- User evaluation}

The hand was tested by able-bodied right-handed male subjects $(n=13$, average age $27 \pm 1$ years). Able-bodied subjects were chosen, instead of amputees, as using amputee subjects would impose an unnecessary burden to the small group of available subjects. Ethical approval for this study was obtained from the institutional ethical committee (file number: \# 1-4-2012). The Delft Cylinder Hand was attached at the palmar side of the left hand (Figure 1), using a Pro Cuff (TRS inc.). A second Pro Cuff was used to create an attachment point for the shoulder harness. To evaluate the functionality of the hand, two tests were selected, the Box and Block Test (BBT) [34, 35] and the Nine Hole Peg Test (NHPT) [36]. Both tests are commonly used to assess hand function, e.g. in patients with stroke $[37,38]$ or Parkinson disease $[39,40]$. There are several studies in the field of upper limb prosthetics which used the BBT to measure functional outcomes [41-44]. Miller et al. identified the BBT as a promising functional evaluation test for upper limb prosthetics [45]. Until now the application of the NHPT for the evaluation of terminal devices is rare [46]. This test was included as it requires more fine motor skills than the BBT.

Both tests can be performed within a few minutes, so multiple trials can be performed in one session. This is a benefit over other test instruments, like the Southampton Hand Assessment Procedure (SHAP) [47, 48], the Jebsen Hand Function Test (JHFT) [49] and the Sollerman Hand Function Test (SHFT) [50]. As these tests take a much longer time to administer (around 15-30 minutes), they are less suitable for many repetitions in a cohort study [51]. The outcomes of the BBT were compared to the outcomes published by Farrell and Weir [43] and the outcomes found by Resnik and Borgia [42]. The results of the NHPT were compared to outcomes 
found by Schabowsky et al. [46]. A commercially available BBT was used and a home-made NHPT (holes: $\varnothing 10 \times 15 \mathrm{~mm}$ with a $32 \mathrm{~mm}$ centre distance, pegs: $\varnothing 9 \times 32 \mathrm{~mm}$ ).

\section{-- Protocol user evaluation}

The subjects performed the tests in three sessions at three different days. Each session consisted of five trials of the BBT, followed by five trials of the NHPT. As the BBT is a simpler task and it takes less time and effort from the user than the NHPT, the BBT was performed first. In this way the mutual influence of the tests was minimized. It was not necessary to randomize the order of the tests, as the tests were not mutually compared. Both tests were executed according to the test instructions of the BBT [34] and the NHPT [36]. Before the test started the test procedure was explained to the subject and an informed consent from the subject was acquired. A basic instruction on how to operate the hand was given to the subject. The subjects were given a minute to get used to operating the hand before the session started.
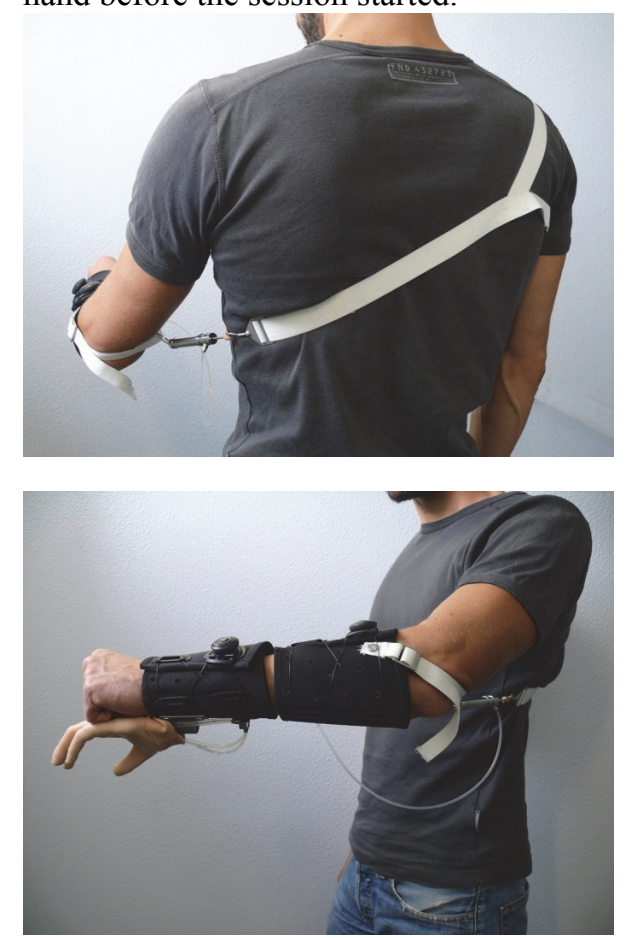

Figure 1 Two pro-cuffs (TRS inc.) were used to attach the Delft Cylinder Hand to a healthy test subject. The hand can be closed by pulling the white shoulder strap, which is attached to the master cylinder.

\section{Amputee opinions}

As a final part of the user evaluation three transradial amputee subjects were allowed to use the Delft Cylinder Hand for several minutes. They were asked to give their comments on the hand. The first subject was a male who was a regular user of a myoelectric prosthesis, the second was a male who was a regular user of both myoelectric and bodypowered hands, the third was a female subject who abandoned the use of a prosthesis, as she considered it to be to heavy and of little use.

\section{RESULTS \\ Hand prototype}

The fingers of the hand use 7 active DoF's that are actuated by miniature hydraulic slave cylinders (S17, Figure 2, Figure 3). The cylinders were specially designed for the hand, to enable a lightweight and compact design. Three cylinders $(\mathrm{D}=8 \mathrm{~mm})$ are located inside the index, middle and ring finger. These cylinders control the PIP-joints. Three other cylinders $(\mathrm{D}=7 \mathrm{~mm})$ control the MCP-joints. These cylinders are located inside the palm of the hand. A seventh cylinder $(\mathrm{D}=7 \mathrm{~mm})$ controls the MCP- and PIP-joint of the little finger by a four bar mechanism. This cylinder is also located in the hand palm. The user controls the slave cylinders by pulling a shoulder strap (Figure 1), which is attached to a master cylinder $(D=10 \mathrm{~mm}, M 1$ in Figure 3 ). The working principle of the hydraulic system (Figure 3) is analogous to the braking system of a car, in which the master cylinder of the brake pedal actuates the slave cylinders at the four wheels. When the user of the prosthesis pulls the shoulder strap, the master cylinder extends and actuates the slave cylinders at the fingers. The fingers of the hand then close. The cylinders of the fingers act like communicating vessels. Therefore the fluid pressure in the master cylinder and in each slave cylinder, are equal to each other at every moment. The pressure in the master cylinder also results in a reaction force at the shoulder harness. This provides the user with proprioceptive force feedback on the grip strength and it provides an indication of the object stiffness. No additional sensors are needed to control the grip force or the finger motion of this underactuated hand. During closing of the hand if one finger is blocked, the other fingers will continue closing. When there is an object present within the hand, the fingers will adapt to its shape. Depending on the shape of the object, the hand will adapt to a pinch grip or a power grip (Figure 4). The users can control the grip force and the degree of hand opening, by exerting an actuation force to the shoulder harness. The hand has a maximum opening span of $75 \mathrm{~mm}$. Recordings with a high-speed camera showed that the hand closed within $250 \mathrm{~ms}$. The hydraulic system could stand a system pressure up to $6 \mathrm{MPa}$ (60 bar). This enables forces up to $300 \mathrm{~N}$ per cylinder and a pinch force exceeding $30 \mathrm{~N}$ per finger, or $60 \mathrm{~N}$ for a tripod grip.

In addition to the 7 active degrees of freedom, the hand has 7 passive degrees of freedom. The hand has a passively opposable thumb (1 DoF), which can be adjusted by using the sound hand. The thumb has no flexing joints. This makes it easier to align the prosthesis with an object, as the tip of the 
This is the author's version of an article that has been published in this journal. Changes were made to this version by the publisher prior to publication. The final version of record is available at http://dx.doi.org/10.1109/TNSRE.2014.2342158

thumb will not move during hand closing. The fingers allow for $+/-8^{\circ}$ passive ad- and abduction (4 DoF's) at the Carpometacarpal (CMC) joints. This enables placement of objects between the fingers and it protects the fingers against side impact. The wrist has $+/-10^{\circ}$ passive flexion/extension ( $\left.1 \mathrm{DoF}\right)$, to reduce compensatory motion and to protect the fingers from palmar or dorsal impact. The hand can be connected to standard wrists, to allow for passive wrist pro- supination (1 DoF).

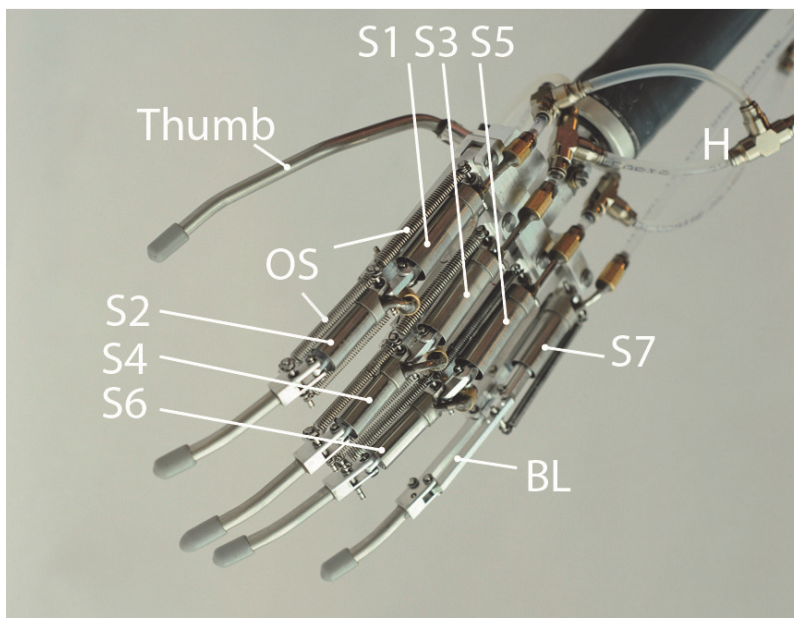

Figure 2 Hand frame of the Delft Cylinder Hand. The index, middle and ring finger have two slave cylinders each (S1-S6), the little finger is actuated by one slave cylinder (S7) combined with a four bar linkage (BL). The hoses $(\mathrm{H})$ connect the slave cylinders to each other and to the master cylinder (see Figure 1), and distribute the hydraulic fluid among the slave cylinders. The fingers flex when the cylinders are actuated. After actuation the fingers are extend by the opening springs at the side of each finger (OS). The thumb is passively opposable.

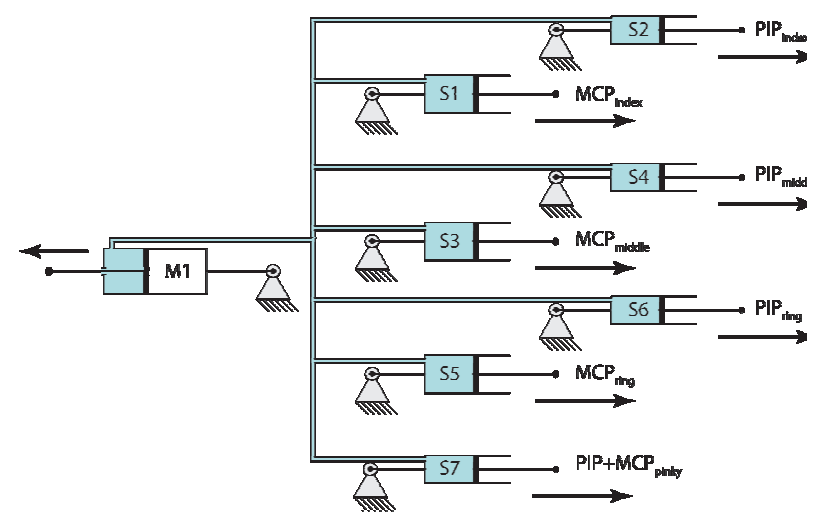

Figure 3 Schematic representation of the hydraulic system. When the master cylinder (M1) is pulled, the slave cylinders (S1-S7) will exert a force at the finger joints. The slave cylinders act like communicating vessels.

The mechanical as well as the hydraulic elements of the hand are fully modular. If instead of one input signal more signals would become available, the hydraulic system can be easily rearranged for multiple DoF control. Finger parts or complete fingers can be easily replaced or reconfigured. Reconfiguring the entire hand from a right-hand to a left-hand version, requires replacement of only two parts from the thumb.

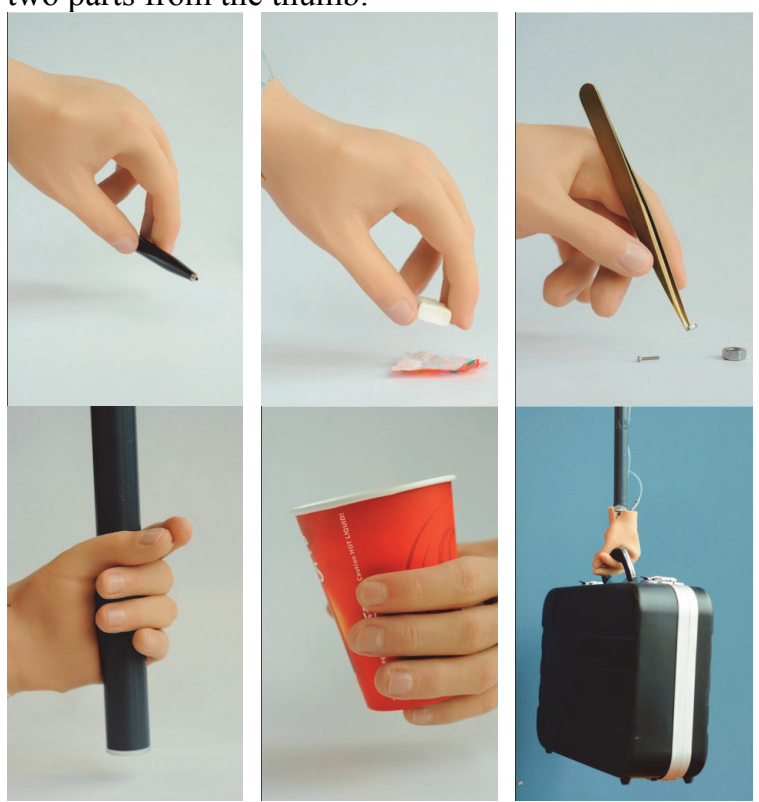

Figure 4 The underactuated hand adapts to the shape of the grasped object, without using any sensor and by using only one control signal. The hand can perform the two basics grasp patterns: precision grip and power grip. The hand can pick up small objects with the precision grip (top left and middle). The feedback allows precision tasks, like handling a pair of tweezers (top right). The hand can hold cylindrical objects with the power grip (below left). The little finger can be used to support the object, e.g. a cup (below middle). The power grip can also be used to carry a load in a horizontal position, e.g. the handlebar of a suitcase (below right).

\section{Hand materials}

The hand and finger frames were made out of aluminium Al7075 T6. The cylinders and axis were made out of stainless steel. After the aluminium was anodized the hand is completely water and dirt resistant. The hand can be covered with a standard sized 71/2" cosmetic glove. The hand frame was covered with foam parts, to protect the cosmetic glove. The hand frame, including the protection foam, has a mass of 152 gram. The total hand including silicone glove has a mass of 217 gram. The master cylinder has a mass of 54 gram. Table I shows an overview of the specifications of the Delft Cylinder Hand, together with the specifications of current available BP hands. 
Table I Specifications of the Delft Cylinder Hand, compared to current available BP hands.

\begin{tabular}{|c|c|c|}
\hline & Current BP hands & $\begin{array}{ll}\text { Delft } & \text { Cylinder } \\
\text { Hand } & \\
\end{array}$ \\
\hline $\begin{array}{l}\text { Cosmetic } \\
\text { appearance }\end{array}$ & $\begin{array}{lr}\begin{array}{l}\text { Cosmetic } \\
\text { adapted } \\
\text { mechanism }\end{array} & \text { to } \\
\end{array}$ & $\begin{array}{l}\text { Mechanism adapted } \\
\text { to an } \\
\text { anthropomorphic } \\
\text { cosmetic glove (size: } \\
7 \frac{1}{\prime \prime}{ }^{\prime \prime} \text { ) }\end{array}$ \\
\hline $\begin{array}{l}\text { Mass entire } \\
\text { hand }\end{array}$ & $\begin{array}{l}\sim 350 \mathrm{~g}, \text { excl. cosmetic } \\
\text { glove } \\
\sim 423 \mathrm{~g}, \text { incl. cosmetic } \\
\text { glove }\end{array}$ & $\begin{array}{l}152 \text { g, excl. } \\
\text { cosmetic glove } \\
217 \text { g, incl. cosmetic } \\
\text { glove }\end{array}$ \\
\hline $\begin{array}{l}\text { Energy } \\
\text { transmission }\end{array}$ & $\begin{array}{l}\text { Bowden cable and } \\
\text { mechanic linkages }\end{array}$ & Hydraulic \\
\hline $\begin{array}{l}\text { Actuation } \\
\text { energy }\end{array}$ & $\sim 1.60-1.70 \mathrm{Nm}$ (or J) & $0.828 \mathrm{Nm}$ (or J) \\
\hline $\begin{array}{l}\text { Maximum } \\
\text { pinch force }\end{array}$ & $\begin{array}{l}\sim 15 \mathrm{~N} \text { precision grip } \\
\text { (or three-jaw chuck) }\end{array}$ & $\begin{array}{l}30 \mathrm{~N} \text { one finger } \\
60 \mathrm{~N} \text { precision grip } \\
\text { (or three-jaw chuck) }\end{array}$ \\
\hline Control & Shoulder control & Shoulder control \\
\hline DoF's & 1 in the entire hand & $\begin{array}{l}2 \text { active per finger } \\
1 \text { active in the little } \\
\text { finger } \\
1 \text { passive per finger } \\
1 \text { passive for the } \\
\text { thumb }\end{array}$ \\
\hline $\begin{array}{l}\text { DoF control in } \\
\text { hand }\end{array}$ & $\begin{array}{l}1 \text { signal }->1 \mathrm{DoF} \\
\text { (fully actuated) }\end{array}$ & $\begin{array}{l}1 \text { signal -> } 7 \text { DoF's } \\
\text { (underactuated) }\end{array}$ \\
\hline $\begin{array}{l}\text { Basic grasp } \\
\text { patterns }\end{array}$ & Pinch/Tip grip & $\begin{array}{l}\text { Pinch/Tip grip } \\
\text { Power grip }\end{array}$ \\
\hline $\begin{array}{l}\text { Other grasp } \\
\text { patterns }\end{array}$ & Tripod grip & $\begin{array}{l}\text { Hook grip } \\
\text { Spherical grip } \\
\text { Extension/Palmar } \\
\text { grip } \\
\text { Tripod grip }\end{array}$ \\
\hline $\begin{array}{l}\text { Maximum } \\
\text { hand opening }\end{array}$ & $\sim 70 \mathrm{~mm}$ & $75 \mathrm{~mm}$ \\
\hline
\end{tabular}

\section{Hand evaluation}

\section{- Mechanical evaluation}

The mechanical performance of the hand was quantified by four different test. The results of the tests were compared to data of current BP hands.

1. In the first test the actuation force and displacements were measured, while the hand opened and closed without pinching. Figure 5 shows an example of a force displacement curve. One cycle is shown, as the deviations between the four cycles were small. For one cycle the hand (with glove and master cylinder) required $0.885 \pm 3 \cdot 10^{-3} \mathrm{Nm}$ (or J) of energy. This energy is represented by the surface between the upper line of the graph and the $\mathrm{x}$-axis, see Figure 5. The hand dissipated $0.642 \pm 3 \cdot 10^{-3} \mathrm{Nm}$ (or J) of energy, which is represented by the surface enclosed by the curve.

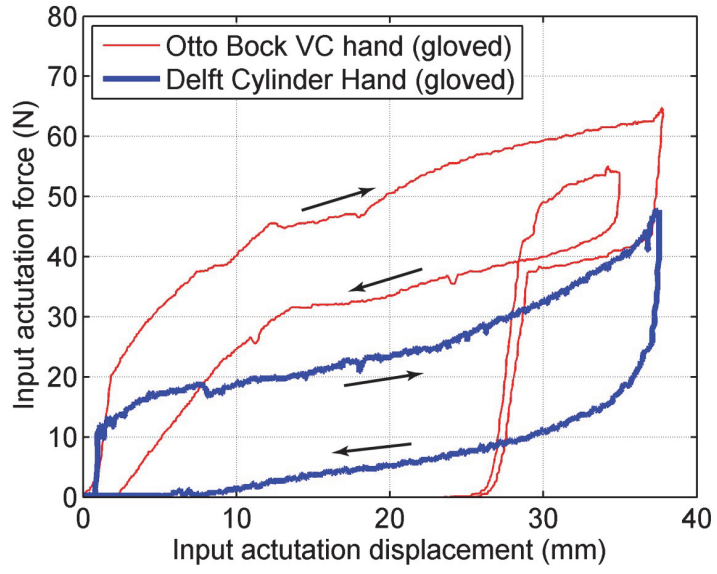

Figure 5 Force displacement curve of the closing test, representing a full cycle of closing and re-opening of the Delft Cylinder Hand (thick blue line). For clarity only one cycle is shown, as the four cycles were very similar. As a reference the curve of the Otto Bock VC hand (thin red line) is shown, adapted from [11]. Note the difference in force level between both hands, and note the small extra loop of the Otto Bock hand, due to its automatic locking mechanism.

2. In the second test the actuation force and displacement were measured, during a full cycle of closing, opening and pinching with a force of $15 \mathrm{~N}$. During one cycle the hand required $0.828 \pm 3 \cdot 10^{-3} \mathrm{Nm}$ (or $\mathrm{J}$ ) of energy. Of this energy $0.546 \pm 3 \cdot 10^{-3} \mathrm{Nm}$ (or J) was dissipated by the mechanism and the cosmetic glove.

3. During the third test the pinch force and the actuation force were measured. Figure 6 shows the pinch force a as a function of an actuation force increasing from 0 to $100 \mathrm{~N}$. At an actuation force of $20 \mathrm{~N}$ the fingers touched the $10 \mathrm{~mm}$ thick load cell and commenced pinching. When the actuation force increased further the pinch force increased linearly. The slope of the linear line is determined by the transmission ratio of the system.

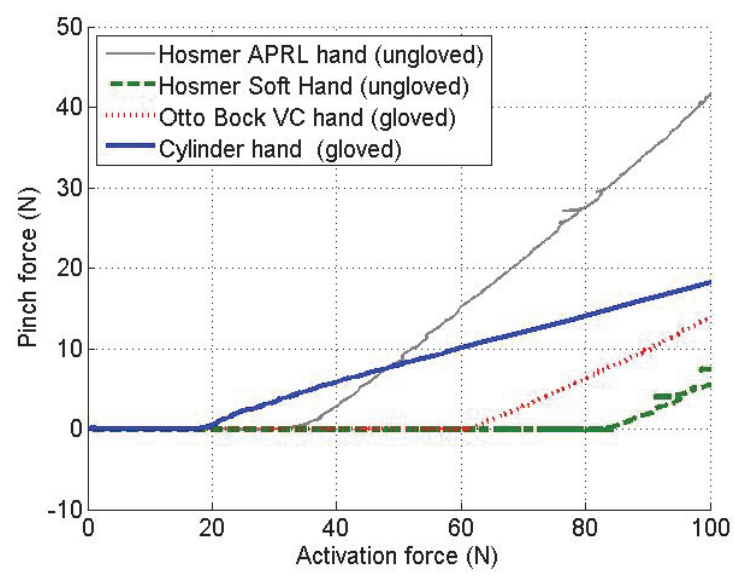

Figure 6 The force displacement curve of the $100 \mathrm{~N}$ actuation test. The graph displays the characteristic of the Delft Cylinder 
Hand together with the diagrams of the ungloved APRL and Hosmer Soft hand and a gloved Otto Bock hand. (Adapted from [11]). The Delft Cylinder hand requires a lower actuation force $(20 \mathrm{~N})$ to start building up pinch force, than the other hands. Its slope can be changed by using another master cylinder, whereas the other hands have a fixed slope.

4. In the endurance test the hand had to close, pinch $15 \mathrm{~N}$ and re-open 100,000 times. During the test, the apparatus paused several times, each time because one of the endpoint conditions was not met. After 33,493 cycles the MCP-springs had to be replaced, when the end loops of the MCPsprings were broken. After 54,696 cycles two MCP-springs had a broken end loop. The MCP-springs were therefore replaced by springs with a thicker wire diameter. After 76,695 cycles the bearings and the O-rings had to be re-lubricated. After 80,330 cycles the hydraulic system had to be refilled. At the end of the test the silicone cosmetic glove was worn out. The test was finished after 100,000 cycles without the failure of parts of the hand frame and without noticeable fluid leakage. No major repairs were required.

\section{- User evaluation}

Box and block test

The number of blocks transferred increased over the length of the trials (Figure 7), from $17 \pm 6$ blocks in the first trial to $26 \pm 8$ blocks in the $15^{\text {th }}$ trial.

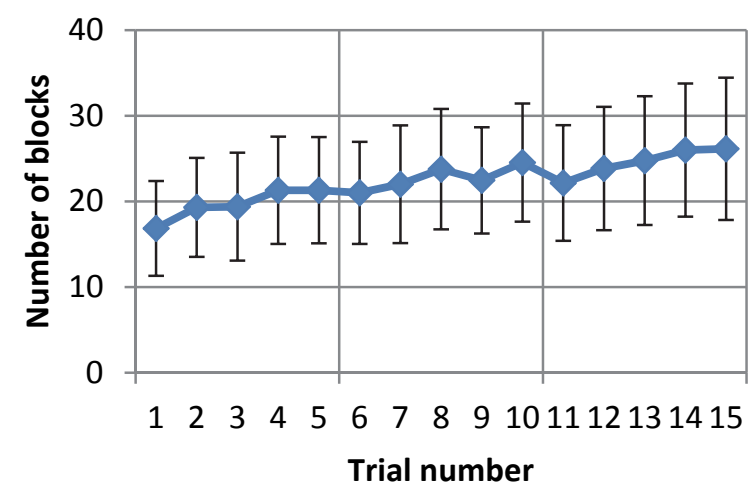

Figure 7 Scores of the Box and Block Test (BBT). The test was performed in three sessions of five trials at three different days $(\mathrm{N}=13, \mathrm{SD}$ over 13 subjects).

\section{NHPT}

The required time to complete the NHPT decreased over the trials (Figure 8), from $117 \pm 41 \mathrm{~s}$ in the first trial to $62 \pm 11 \mathrm{~s}$ in the $15^{\text {th }}$ trial.

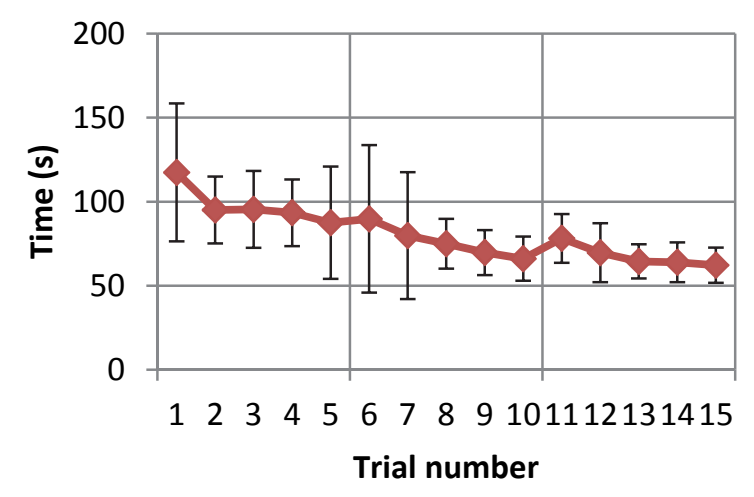

Figure 8 Scores of the Nine Hole Peg Test (NHPT). The test was performed in three sessions of five trials at three different days ( $N=13$, SD over 13 subjects).

\section{Amputee opinions}

The amputee subjects that tried the prosthesis were all enthusiast about the hand and commented that it was easy to operate and that is was remarkable light, in comparison to their current and past prostheses.

\section{DISCUSSION \\ Mechanical evaluation}

- Actuation energy

Although the Delft Cylinder Hand has more joints and a larger range of motion, it required less input energy for closing $\left(0.885 \pm 3 \cdot 10^{-3} \mathrm{Nm}\right.$ or $\left.\mathrm{J}\right)$ than other body-powered VC hands, e.g. the Otto Bock VC hand $\left(1.639 \pm 24 \cdot 10^{-3} \mathrm{Nm}\right)$, the APRL hand $\left(1.058 \pm 4 \cdot 10^{-3} \mathrm{Nm}\right)$ and the Hosmer Soft VC hand $\left(2.292 \pm 12 \cdot 10^{-3} \mathrm{Nm}\right)$ [11]. This means that the user has to deliver less input energy to operate the hand. Grasping and pinching an object with $15 \mathrm{~N}$ required less energy $\left(0.828 \pm 3 \cdot 10^{-3} \mathrm{Nm}\right)$ than fully closing all fingers, because pinching an object requires a smaller joint motion of the index and the middle finger. The required energy for closing and pinching was less than that of the Otto Bock VC hand $\left(1.694 \pm 16 \cdot 10^{-3} \mathrm{Nm}\right)$, and the Hosmer Soft VC hand $\left(2.176 \pm 16 \cdot 10^{-3} \mathrm{Nm}\right)$ and it was similar to the APRL hand $\left(0.831 \pm 1 \cdot 10^{-3} \mathrm{Nm}\right)$ [11]. It should be noted that the APRL hand, and the other hands, were tested without a Bowden cable transmission and without a cosmetic glove, which both will require considerable (10-40\%) extra input energy. All current BP prosthesis use a Bowden-cable transmission, to transmit the force form the harness to the hand. The Delft Cylinder Hand uses a hydraulic transmission, instead of a Bowden-cable. The hand was tested with a hydraulic transmission and with a cosmetic glove applied.

- Actuation force

The Delft Cylinder Hand required an actuation force of only $20 \mathrm{~N}$ to grasp a $10 \mathrm{~mm}$ thick object 
and start pinching. This actuation force is $15-65 \mathrm{~N}$ lower than that of other body-powered VC hands (Figure 6). The Delft Cylinder Hand has a flatter slope than the other hands, because of its lower transmission ratio. This ratio can however easily be changed, by using a master cylinder with a smaller or larger diameter. A cylinder with a smaller diameter will increase the slope. In this way the transmission ratio can be adapted to individual preferences. Another option is to add a dual phase mechanism which can switch between two transmission ratios $[52,53]$. This principle can relatively easy be implemented in hydraulics [54], to increase the pinch force at a low actuation force. Its low required input energy and its low required actuation force are a major benefit over current hands, and make the Delft Cylinder Hand also suitable for people with less body strength.

\section{- Endurance}

The hand passed the endurance test as it did not require major repair and showed no hydraulic leakage. A user who uses his/her hand prosthesis on average 274 times a day, would have to use the prosthesis for one year to reach 100,000 cycles. The hand required only small maintenance during the test. At $75 \%$ of the test the hand had to be relubricated and at $80 \%$ of the test the hydraulics had to be refilled. Although there was no fluid leakage, the fluid could slowly disappear from the system, as during every stroke some fluid could evaporate from the wetted cylinder surface. When 100,000 cycles equal one year, 75,000-80,000 cycles would correspond to 8-9 months. This is an acceptable interval for small maintenance, as the small maintenance can easily be done by the prosthetist when the cosmetic glove is replaced (typically every 3-6 months). Furthermore the test revealed the sensitivity to fatigue of the end loops of the MCP-springs. This can be solved by replacing the springs by springs with a larger wire diameter, as was done during the test, or by using a helical spring with an alternative and stronger end loop.

\section{User evaluation}

In the Box and Block Test as well as in the Nine Hole Peg Test the users were able to use their prosthesis directly after donning and they all were able to complete the tests on their first trial. The subjects had been given only a basic instruction and a minute to get used to the prosthetic hand. This illustrates that the control is intuitive and easy to learn. A clear trend can be observed in both tests (Figure 7, Figure 8). Over the trials the performance of the subjects increased. At the start of each new session the users performed less well than at the end of the previous session. During each session the performance increased gradually.
The results of the functional tests were compared to BBT-scores found in literature. Farrell and Weir [43] found BBT-scores of $25 \pm 3.5$ blocks for slow devices and $28 \pm 4$ blocks for fast devices, in a study with able-bodied subjects $(n=20)$ using myoelectric terminal devices. Resnik and Borgia [42] found BBT-scores of $19.9 \pm 10.0$ blocks for transradial amputees $(n=26)$, who were full-time or part-time users of myoelectric or body-powered terminal devices. The scores of these studies are similar to the BBT-scores found in our study $(26 \pm 8$ blocks, during the $15^{\text {th }}$ trial). It should however be noted that the scores of the Delft Cylinder Hand might still improve after the $15^{\text {th }}$ trial. Furthermore the tests were performed by right-handed subjects, using a left-hand prosthesis. The tests results might improve when the tests would be performed by lefthanded subjects, or by using a right-hand version for right-handed subjects.

The results of the NHPT illustrate the capability to perform fine motor tasks using the Delft Cylinder Hand. The values can serve as a quantitative measure for comparison to other prosthetic hands. In a study by Schabowsky et al. [46] skilled transradial amputees ( $n=6$, mean age: $50.2 \pm 14$ years) finished the NHPT in $76 \pm 29 \mathrm{~s}$, by using their bodypowered hook. This reference data suggests that the functional performance of Delft Cylinder Hand in the NHPT $(62 \pm 11 \mathrm{~s})$ was at least similar to the score of a BP-hook. This is a very interesting result, given the fact that the BP-hook is considered to be still one of the most functional terminal devices. More reference data is needed to support these findings, as Schabowsky et al. only measured six subjects.

\section{Evaluation requirements \\ - Cosmetic appearance}

The hand mechanism fits inside a standard 7 1/2" size anthropomorphic cosmetic glove, made of Silicone or PVC. These gloves, which are normally used for passive cosmetic hands, are moulded copies of a human hand. As the shape of the mechanism was adapted to the shape of the glove, the hand has the exact anthropomorphic dimensions of a human hand.

\section{- Mass}

According to the study by Biddiss et al. the development of a more light-weight comfortable prosthesis is the most important design priority [6]. Other studies also report the importance of weight reduction in hand prostheses [55-57]. Fishman and Berger [9] already reported in 1955 that the APRL hand (347 gram, without a glove) was too heavy to be fitted to amputees with short or weak residuals. Despite these reports, the most recently developed hands are considerably heavier than the APRL hand; i-limb ultra (469 gram, without a glove), bebionic v3 (550 gram, without a glove) and 


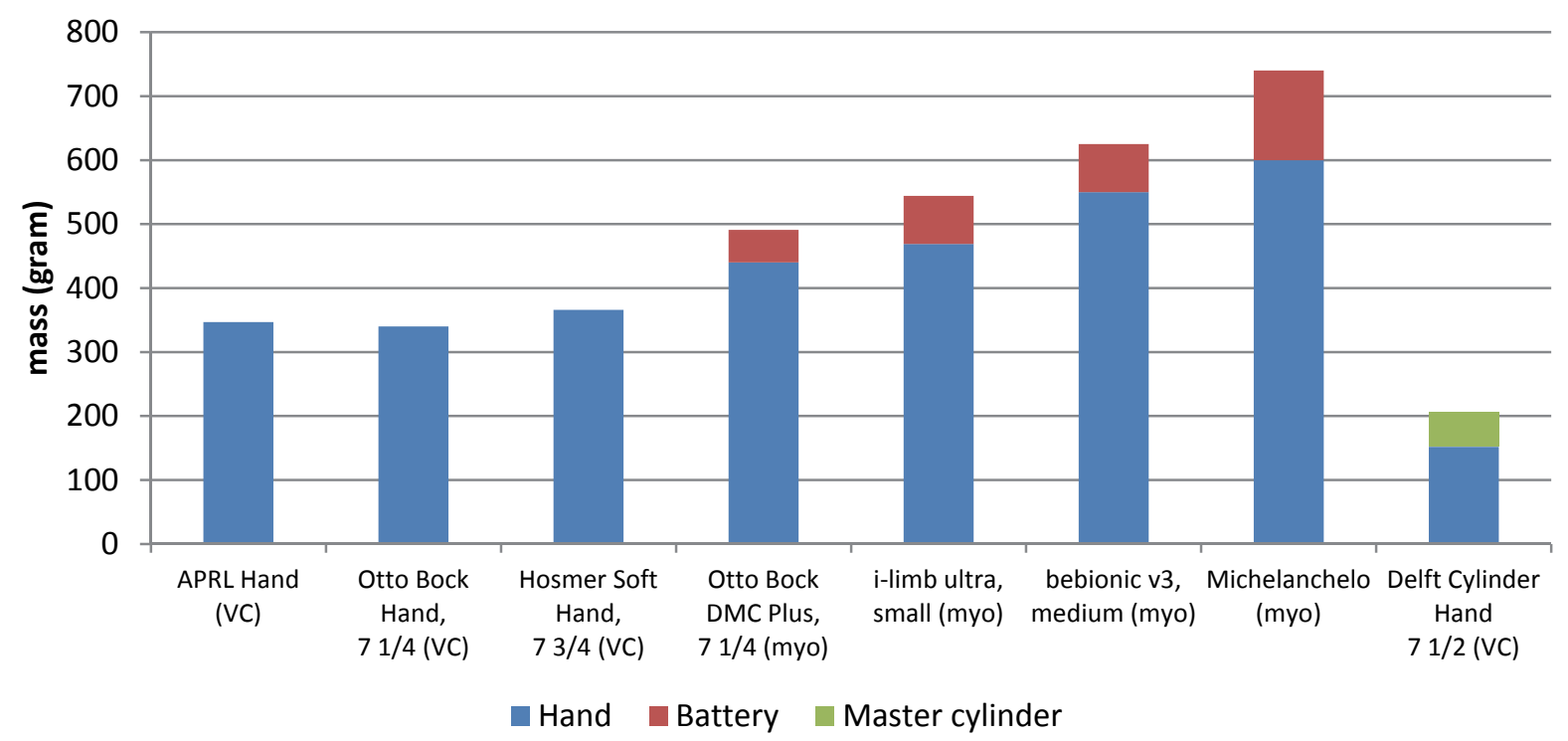

Figure 9 The mass of the Delft Cylinder Hand (right) compared to current state-of-the-art commercially available body-powered voluntary closing hands (VC) and myoelectric hands (myo). (All hand masses are without the cosmetic glove). The figure also shows the battery masses of the Myo-electric hands.

Michelangelo (600 gram, without a glove). Figure 9 shows the mass of current commercially available prosthetic hands (347-600 gram, without a glove), compared to the mass of the Delft Cylinder Hand (152 gram, without glove). The Delft Cylinder Hand is $55 \%$ lighter than the lightest current VC hand and $68 \%$ lighter than the lightest current articulating hand. The low mass of the Delft Cylinder hand has the potential to improve wearing comfort and will enable more amputees to wear a prosthetic hand, e.g. amputees with a short or weak residual arm.

\section{- Actuation energy and pinch force}

The application of a hydraulic transmission enabled an efficient transmission and distribution of the actuation energy to the multiple finger joints, without additional friction forces. In this way the force feedback information is optimized and the input force is reduced. This reduces harness discomfort, which is one of the main problems associated with body-powered operation [6]. The elastic part of the required input energy was minimized, by making the system stiff, by choosing springs that were not stronger than necessary and by using a silicone glove, instead one made of PVC [22]. The resulting part of the required input energy consists mainly of hysteresis. The hysteresis was reduced by minimizing the bearing and the sealing friction. The Delft Cylinder Hand, which has 7 DoF's, required less energy than current body powered hands which have only one DoF. The hand could deliver a pinch force of over $30 \mathrm{~N}$ per finger, and $60 \mathrm{~N}$ for a tripod grip, which exceeds the requirements.

\section{- Control of the hand}

The application of body-powered control in the Delft Cylinder hand, offers the user a prosthesis that has a low mass, provides force and position feedback, and that can be operated without unwanted hand movements, at a high speed and without additional noise. This is an advantage over myoelectric prostheses. Beside a high mass, users of electric devices report a lack of feedback $[5,56$, 57], unwanted hand movement [56], a slow closing speed [55-57] and a disturbing motor noise [56, 57]. By using body-powered actuation these problems were avoided. The shoulder strap of the Delft Cylinder Hand offers intuitive force and position feedback to the user, based on the principle of Extended Physiological Proprioception (EPP) [58]. It enables easy and accurate control of the hand and avoids undesired opening or closing of the hand. The entire hand can be fully closed within $<250 \mathrm{~ms}$, which approaches the speed of a human hand $(\sim 100 \mathrm{~ms})$. Electric hands require a minimum closing time ranging from $333 \mathrm{~ms}$ (Vari Plus Speed [59]) to $1200 \mathrm{~ms}$ (i-limb ultra [60]) and they require an additional delay ranging from $50 \mathrm{~ms}$ to over $300 \mathrm{~ms}$ [43], to process the myoelectric signal in the microcontroller. Unlike electric hands the Delft Cylinder Hand is completely silent, which avoids undesired attention.

\section{- Articulating fingers}

The hand has articulating fingers, which can perform both pinch and power grasp. The underactuated fingers adapt to the shape of the grasped object. This enables stable grasping of a broad range of objects, without the need of extra sensors. 


\section{- Environmental influences}

The hand is corrosion resistant and does not use electronic parts. The hand can function in wet and dirty environment, which enables a broad application of the hand.

\section{- Modular system}

The hand is fully modular. The finger parts or complete fingers can be easily replaced or reconfigured. All parts fit inside the hand, making the Delft Cylinder Hand also applicable for amputees with very long arm residuals or a transcarpal hand amputation. The transmission-ratio can be adapted to the individual patient by changing the master cylinder. These factors give the prosthetist the ability to adapt the hand to many different individual users with totally different needs. For example: people with very high amputations or very long residua, or people who have a low strength (including elderly users).

\section{Clinical implications}

The current prosthesis options do not meet all basic user demands, cosmetics, control and comfort, simultaneously. BP hooks are fast, accurate, and they have a low mass. However they have a poor cosmetic appearance. BP hands have a reasonable appearance, however they are uncomfortable to operate and have a poor function. The myoelectric hands combine function and a reasonable appearance. However they are slow, noisy, they lack force feedback and according to many users they are too heavy to be worn comfortably. The Delft Cylinder Hands is the first hand that combines the basic user demands in one device. The hand has a cosmetic anthropomorphic appearance, static as well as kinematic. It could be easily controlled with little familiarity and it performed at least similar to electric devices and BP-hooks. The hand is fast and accurate, due to the proprioceptive feedback. It is comfortable to operate and to wear, due to the low actuation force and its very low mass. The adaptive fingers enable the hand to perform the basic grasps, enabling the user to use the hand in many different activities of daily living. Combining the basic user demands in one device, makes the Delft Cylinder Hand a promising alternative to current available devices.

\section{Study strengths and limitations}

A strength of this study is the quantitative functional evaluation of the prototype. Most studies that describe prosthesis prototypes do not provide quantitative outcomes, which makes it impossible to objectively compare them to other devices. A limitation of this study was that right-handed subjects were tested, using a left-hand device. This could have influenced the results negatively. Also the prosthesis was only tested inside the laboratory. Extensive clinical testing is required, to see how the hand performs in daily living situations outside the laboratory. Therefore as a next step the prosthesis will be provided to a group of amputees, who will be able to use the prosthesis at home. This test is planned to start within a year. The home trial might also find an answer to the practical questions, e.g. whether it is desirable to add a locking mechanism to the hand, which can be used for holding objects for a longer time.

\section{CONCLUSIONS}

This study presents the Delft Cylinder Hand, a prosthetic hand prototype that is anthropomorphic, slender, fast and silent. The hand meets one of the most important user design criteria, which is a low hand mass. Its mass (152 gram without glove; 217 gram with glove) is $68 \%$ lower than the lightest available articulating myoelectric hand and 55\% lighter than the lightest BP hand of similar size. The hand has articulating fingers which fully adapt to the grasped object, by using the principle of underactuation. Its body-powered actuation provides the user with proprioceptive force and position feedback, enabling accurate and fast control, without the need of additional sensors. In functional tests (Box and Block Test and Nine Hole Peg Test) the hand showed scores that were at least similar to current body-powered hooks and myoelectric hands. Through the application of a hydraulic cylinder transmission, the hand required $49-162 \%$ less energy from the user than current body-powered hands and it had a higher maximum pinch force $(30-60 \mathrm{~N})$. Its very low mass, its anthropomorphic shape and kinematics, and high functional scores, make this hand prototype the first prosthetic hand that meets the basic user requirements.

\section{REFERENCES}

[1] D. S. Childress, "Historical aspects of powered limb prostheses," Clinical Prosthetics and Orthotics, vol. 9, pp. 2-12, 1985.

[2] H. K. Bowker and J. W. Michael, Eds., Atlas of Limb Prosthetics: Surgical, Prosthetic, and Rehabilitation Principles.: Rosemont, IL, American Academy of Orthopedic Surgeons, 1992, p.^pp. Pages.

[3] R. H. Meier and D. J. Atkins, Functional restoration of adults and children with upper extremity amputation. New york, N.Y.: Demos Medical Publishing, Inc., 2004.

[4] D. H. Plettenburg, Upper Extremity Prosthetics: Current status \& evaluation. Delft: VSSD, 2006.

[5] E. Biddiss and T. Chau, "Upper-limb prosthetics: Critical factors in device abandonment," American Journal of Physical Medicine and Rehabilitation, vol. 86, pp. 977987, 2007

[6] E. Biddiss, D. Beaton, and T. Chau, "Consumer design priorities for upper limb prosthetics," Disability and Rehabilitation: Assistive Technology, vol. 2, pp. 346-357, 2007.

[7] D. H. Plettenburg, "Basic requirements for upper extremity prostheses: The Wilmer approach," presented at the 20th Annual International Conference of the IEEE Engineering in Medicine and Biology - Proceedings, Hong Kong, China, 1998. 
[8] O. Niet, van der, H. A. Reinders-Messelink, R. M. Bongers, H. Bouwsema, and C. K. Sluis, van der, "The iLIMB hand and the DMC plus hand compared: A case report," Prosthetics and Orthotics International, vol. 34, pp. 216-220, 2010.

[9] S. Fishman and N. Berger, "The choice of terminal devices," Artificial Limbs, vol. 2, pp. 66-77, May 1955.

[10] S. G. Millstein, H. Heger, and G. A. Hunter, "Prosthetic use in adult upper limb amputees: A comparison of the body powered and electrically powered prostheses," Prosthetics and Orthotics International, vol. 10, pp. 2734, 1986.

[11] G. Smit and D. H. Plettenburg, "Efficiency of voluntary closing hand and hook prostheses," Prosthetics and Orthotics International, vol. 34, pp. 411-427, 2010.

[12] G. Smit, R. M. Bongers, C. K. Sluis, van der, and D. H. Plettenburg, "Efficiency of voluntary opening hand and hook prosthetic devices, 24 years of development?," Journal of Rehabilitation Research and Development, vol. 49, pp. 523-534, 2012.

[13] S. Schulz, C. Pylatiuk, M. Reischl, J. Martin, R. Mikut, and G. Bretthauer, "A hydraulically driven multifunctional prosthetic hand," Robotica, vol. 23, pp. 293-299, 2005.

[14] F. Janovsky and H. Merten, "Electrohydraulical transmission system for artificial hands (Elektrohydraulisches Antriebssystem für künstlich Hande)," Medizinische Technik, vol. 87, 1967.

[15] D. R. Broome, B. L. Davies, and M. Lord, "A total hydraulically powered prosthetic arm system," Engineering in Medicine, vol. 3, pp. 8-14, 1974.

[16] F. Witte, "Development problems of a electrohydraulic artificial arm for bi-lateral amputees

(Entwicklungsprobleme an einem elktrohydraulischen Kunstarm fuer Ohnarmer)," VDI-Berichte, 1979.

[17] W. Tobergte, "Hydrostatic Converter with direct changeable gear to drive a prosthetic arm (Hydrostatischer Wandler mit stufenlos veränderlicher Übersetzung zum Antrieb einer Armprothese)," VDI-Berichte, pp. 205-211, 1980 .

[18] I. Kato, "Multifuntional myoelectric hand prosthesis with pressure sensory feedback system, Waseda Hand 4P," presented at the 3rd International Symposium on External Control of Human Extremities ETAN, Dubrovnik, 1970.

[19] A. Kargov, C. Pylatiuk, R. Oberle, H. Klosek, T. Werner, W. Roessler, and S. Schulz, "Development of a multifunctional cosmetic prosthetic hand," presented at the IEEE 10th International Conference on Rehabilitation Robotics, ICORR'07, Noordwijk, 2007.

[20] H. Goller and D. W. Lewis, "Hydraulic body-powered system for prosthetic devices," Bulletin of Prosthetics Research, pp. 156-166, 1969.

[21] M. LeBlanc, "Current evaluation of hydraulics to replace the cable force transmission system for body-powered upper-limb prostheses," Assistive Technology: The official journal of RESNA, vol. 2, pp. 101-107, 1990.

[22] G. Smit and D. H. Plettenburg, "Comparison of mechanical properties of silicone and PVC cosmetic gloves for articulating hand prostheses," Journal of Rehabilitation Research and Development, vol. 50, 2013.

[23] R. F. Chandler, C. E. Clauser, J. T. McConville, H. M. Reynolds, and J. W. Young, "Investigation of inertial properties of the human body," Aerospace Medical Research Laboratory, Wright-Patterson AFB DOT HS801 430, March 1975.

[24] A. D. Keller, C. L. Taylor, and V. Zahm, "Studies to determine the functional requirements for hand and arm prosthesis," Department of Engineering, University of California, Los Angeles 1947.

[25] C. L. Taylor, "The biomechanics of the normal and of the amputated upper extremity," in Human limbs and their substitutes, P. E. Klopsteg and P. D. Wilson, Eds., ed New York: McGraw-Hill, 1954, pp. 169-221.

[26] B. Radocy, "Voluntary closing control: a successful new design approach to an old concept," Clinical Prosthetics and Orthotics, vol. 10, pp. 82-86, 1986.
[27] D. H. Plettenburg and J. L. Herder, "Voluntary closing: A promising opening in hand prosthetics," Technology and Disability, vol. 15, pp. 85-94, 2003.

[28] J. R. Napier, "The prehensile movements of the human hand," The Journal of Bone and Joint Surgery, vol. 38 B, pp. 902-913, November 19561956.

[29] D. W. Lamb and K. Kuczynski, The practice of hand surgery: Blackwell, 1981.

[30] T. Laliberté and C. M. Gosselin, "Simulation and design of underactuated mechanical hands," Mechanism and Machine Theory, vol. 33, pp. 39-57, 1998.

[31] L. Birglen, C. Gosselin, and T. Laliberté, Underactuated robotic hands. Berlin: Springer, 2008.

[32] G. A. Kragten, J. L. Herder, and F. C. T. v. d. Helm, "Design guidelines for a large grasp range in underactuated hands," Mechanism and Machine Theory, 2010.

[33] D. J. Gow, W. Douglas, C. Geggie, E. Monteith, and D. Stewart, "The development of the Edinburgh modular arm system," Proceedings of the Institution of Mechanical Engineers, Part H: Journal of Engineering in Medicine, vol. 215, pp. 291-298, 2001.

[34] V. Mathiowetz, G. Volland, N. Kashman, and K. Weber, "Adult norms for the Box and Block Test of manual dexterity," The American journal of occupational therapy. : official publication of the American Occupational Therapy Association, vol. 39, pp. 386-391, 1985.

[35] J. Desrosiers, G. Bravo, R. Hebert, E. Dutil, and L. Mercier, "Validation of the Box and Block Test as a measure of dexterity of elderly people: Reliability, validity, and norms studies," Archives of Physical Medicine and Rehabilitation, vol. 75, pp. 751-755, 1994.

[36] V. Mathiowetz, K. Weber, N. Kashman, and G. Volland, "Adult norms for the Nine Hole Peg Test of finger dexterity," Occupational Therapy Journal of Research, vol. 5, pp. 24-38, 1985.

[37] A. Heller, D. T. Wade, and V. A. Wood, "Arm function after stroke: Measurement and recovery over the first three months," Journal of Neurology Neurosurgery and Psychiatry, vol. 50, pp. 714-719, 1987.

[38] J. H. Cauraugh and S. B. Kim, "Chronic stroke motor recovery: duration of active neuromuscular stimulation," Journal of the Neurological Sciences, vol. 215, pp. 13-19, 2003.

[39] G. M. Earhart, J. T. Cavanaugh, T. Ellis, M. P. Ford, K. B. Foreman, and L. Dibble, "The 9-hole peg test of upper extremity function: Average values, test-retest reliability, and factors contributing to performance in people with parkinson disease," Journal of Neurologic Physical Therapy, vol. 35, pp. 157-163, 2011.

[40] K. S. Lee, W. H. Lee, and S. Hwang, "Modified constraint-induced movement therapy improves fine and gross motor performance of the upper limb in Parkinson disease," American Journal of Physical Medicine and Rehabilitation, vol. 90, pp. 380-386, 2011.

[41] T. A. Kuiken, L. A. Miller, R. D. Lipschutz, K. A. Stubblefield, and G. A. Dumanian, "Prosthetic command signals following targeted hyper-reinnervation nerve transfer surgery," presented at the IEEE-EMBS 2005 27th Annual International Conference of the Engineering in Medicine and Biology Society, Shanghai, 2005.

[42] L. Resnik and M. Borgia, "Reliability and validity of outcome measures for upper limb amputation," Journal of Prosthetics and Orthotics, vol. 24, pp. 192-201, 2012.

[43] T. R. Farrell and R. F. Weir, "The optimal controller delay for myoelectric prostheses," IEEE transactions on neural systems and rehabilitation engineering, vol. 15, pp. 111$118,2007$.

[44] J. S. Hebert and J. Lewicke, "Case report of modified Box and Blocks test with motion capture to measure prosthetic function," Journal of Rehabilitation Research and Development, vol. 49, pp. 1163-74, Dec 2012.

[45] L. A. Miller and S. Swanson, "Summary and recommendations of the academy's state of the science conference on upper limb prosthetic outcome measures," 
Journal of Prosthetics and Orthotics, vol. 21, pp. P83P89, 2009

[46] C. N. Schabowsky, A. W. Dromerick, R. J. Holley, B. Monroe, and P. S. Lum, "Trans-radial upper extremity amputees are capable of adapting to a novel dynamic environment," Experimental Brain Research, vol. 188, pp. 589-601, 2008

[47] C. M. Light, P. H. Chappell, and P. J. Kyberd, "Establishing a standardized clinical assessment tool of pathologic and prosthetic hand function: Normative data, reliability, and validity," Archives of Physical Medicine and Rehabilitation, vol. 83, pp. 776-783, 2002.

[48] P. J. Kyberd, A. Murgia, M. Gasson, T. Tjerks, C. Metcalf, P. H. Chappell, K. Warwick, S. E. M. Lawson, and T. Barnhill, "Case studies to demonstrate the range of applications of the Southampton Hand Assessment Procedure," British Journal of Occupational Therapy, vol. 72, pp. 212-218, 2009.

[49] R. H. Jebsen, N. Taylor, R. B. Trieschmann, M. J. Trotter, and L. A. Howard, "An objective and standardized test of hand function," Archives of Physical Medicine and Rehabilitation, vol. 50, pp. 311-319, 1969.

[50] C. Sollerman and A. Ejeskar, "Sollerman hand function test: A standardised method and its use in tetraplegic patients," Scandinavian Journal of Plastic and Reconstructive Surgery and Hand Surgery, vol. 29, pp. 167-176, 1995

[51] P. J. Kyberd, "The influence of control format and hand design in single axis myoelectric hands: Assessment of functionality of prosthetic hands using the Southampton Hand Assessment Procedure," Prosthetics and Orthotics International, vol. 35, pp. 285-293, 2011.

[52] D. D. Frey and L. E. Carlson, "A body powered prehensor with variable mechanical advantage," Prosthetics and Orthotics International, vol. 18, pp. 118-123, 1994.

[53] J. Kruit and J. C. Cool, "Body-powered hand prosthesis with low operating power for children," Journal of Medical Engineering and Technology, vol. 13, pp. 129133, 13 April 1988 through 15 April 19881989.

[54] J. Van Frankenhuyzen, "Hydraulic brake-system for a bicycle," The Netherlands Patent WO2007111510A1, 2007.

[55] G. H. Kejlaa, "Consumer concerns and the functional value of prostheses to upper limb amputees," Prosthetics and Orthotics International, vol. 17, pp. 157-163, 1993.

[56] P. J. Kyberd, C. Wartenberg, L. Sandsjö, S. Jönsson, D. Gow, J. Frid, C. Almström, and L. Sperling, "Survey of upper-extremity prosthesis users in Sweden and the United Kingdom," Journal of Prosthetics and Orthotics, vol. 19, pp. 55-62, 2007.

[57] C. Pylatiuk, S. Schulz, and L. Döderlein, "Results of an internet survey of myoelectric prosthetic hand users," Prosthetics and Orthotics International, vol. 31, pp. 362370, 2007.

[58] D. C. Simpson, "The choice of control system for multimovement prostheses: Extended Physiological Proprioception (EPP)," in The Control of Upper-Extremity Prostheses and Orthoses, P. Herberts, R. Kadefors, R. Magnusson, and I. Petersen, Eds., ed Springfield, IL: Thomas, 1974, pp. 146-150.

[59] Otto Bock HealthCare. (2011, 12-12-2011). Upper Extremity Prosthetics Catalog. Available: http://www.ottobock.com

[60] Touch Bionics. (2012, i-Limb Ultra, Data Sheet. [Data Sheet]. Available: www.touchbionics.com

\section{ACKNOWLEDGEMENTS}

We would like to thank John Dukker for manufacturing the parts of the hand. We would like to thank the test subjects for participating in the study. Finally we would like to thank Peter Kyberd and Corry van der Sluis for proofreading the paper and for their useful comments.

\section{BIOGRAPHY}

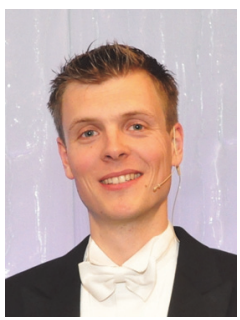

G. Smit received his B Eng degree from the NHL University of Applied Sciences, Leeuwarden, The Netherlands (2005). He received his MSc in Biomechanical Engineering from the University of Twente, Enschede, The Netherlands (2008) and the Ph.D. degree in mechanical engineering design from Delft University of Technology, The Netherlands (2013). He is currently a postdoctoral researcher at the Delft, at the BioMechanical Engineering Department of the Delft University of Technology. His research interests include upper limb prosthetics, underactuated grasping and rehabilitation technology.

Dr. Smit is a member of the International Society for Prosthetics and Orthotics.

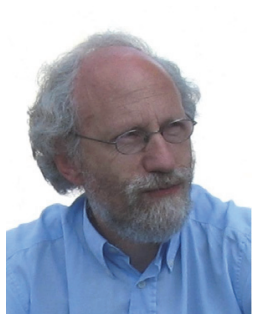

Dick. H. Plettenburg (M'05) received the B.Sc. and M.Sc. degrees in mechanical engineering from University of Twente, The Netherlands, and the $\mathrm{Ph} . \mathrm{D}$. degree in mechanical engineering design from Delft University of Technology, The Netherlands.

$\mathrm{He}$ is currently assistant professor at the BioMechanical Engineering Department of the Delft University of Technology, director of the Delft Institute of Prosthetics and Orthotics, coordinator for the Biomedical Engineering educational programs of Delft University of Technology, and a member of several rehabilitation treatment teams in the Netherlands. His research interests include mechanical engineering design, upper extremity prosthetics, and miniature pneumatics.

Dr. Plettenburg is a member of the International Society for Prosthetics and Orthotics and is Associate Editor of Prosthetics and Orthotics International.



Prof. dr. Frans C. T. van der Helm is professor in Biomechatronics and Biorobotics, Delft University of Technology, and also adjunct-professor at the University of Twente, at Northwestern University in Chicago and Case Western Reserve University, Cleveland. $\mathrm{He}$ has a MSc in Human Movement Science (1985), and $\mathrm{a} \mathrm{PhD} \mathrm{in} \mathrm{Mechanical} \mathrm{Engineering} \mathrm{(1991).} \mathrm{He} \mathrm{was} \mathrm{member}$ of the board of the International Society of Biomechanics (20052009), and participated in the board of the Technical Group of Computer Simulation (TGCS) and the International Shoulder Group (ISG). He is one of programme leaders in the Medical Delta, the collaboration between Leiden Unversity Medical Center (LUMC), Erasmus Medical Center Rotterdam and TU Delft. He is Principal Investigator in the NeuroSIPE (System Identification and Parameter Estimation in Neurophysiological systems) program and H-Haptics (Human centered Haptics) program, sponsored by the Dutch National Science Foundation. In 2012 he received an ERC grant for a research project '4D EEG', improving temporal and spatial resolution of EEG source localization. In 2012 Prof. van der Helm was awarded the Simon Stevin Meester prize, the most prestigious award for technical scientific research in the Netherlands. He has published over 150 papers in international journals on topics as biomechanics of the upper and lower extremity, neuromuscular control, eye biomechanics, pelvic floor biomechanics, human motion control, posture stability, etc. 